\title{
Optimized conditions for gas light interaction in photonic crystal fibres
}

\author{
I. Dicaire*, J-C. Beugnot, L. Thévenaz \\ Swiss Federal Institute of Technology, École Polytechnique Fédérale de Lausanne (EPFL), \\ Institute of Electrical Engineering, Station 11, 1015 Lausanne, Switzerland
}

\begin{abstract}
This paper presents helpful expressions predicting the filling time of gaseous species inside photonic crystal fibres. Based on the theory of diffusion, our gas-filling model can be applied to any given fibre geometry or length by calculating diffusion coefficients. This was experimentally validated by monitoring the filling process of acetylene gas in several fibre samples of various geometries and lengths. The measured filling times agree well within $\pm 15 \%$ with the predicted values for all fibre samples. In addition the pressure dependence of the diffusion coefficient was experimentally verified by filling a given fibre sample with acetylene gas at various pressures. Finally ideal conditions for gas light interaction are determined to ensure optimal efficiency of the sensor by considering the gas flow dynamics in the design of microstructured fibres for gas detection and all-fibre gas cell applications.
\end{abstract}

Keywords: Fibre optics sensors, microstructured devices, absorption, evanescent-field sensor, photonic crystal fibres.

\section{INTRODUCTION}

Photonic crystal fibres (PCFs) are now widely used to study the interaction between light and matter. The large interaction length that is obtained in such small volumes provides efficient gas sensing capabilities. Combined with the ability to engineer their physical and optical properties, photonic crystal fibres are attractive candidates for realizing chemical sensors and gas cells. For instance, solid-core microstructured fibres can be designed to have specific dispersion characteristics or to have maximum overlap between the optical field and the gas species ${ }^{1}$. Applications such as acetylene gas spectroscopy and liquid chemical sensing have been successfully performed in suspended-core fibres where up to $20 \%$ of the optical power is available for sensing via the evanescent field ${ }^{2}$. On the other hand, hollow-core fibres are usually preferred for weak absorption lines or short fibre lengths even considering their limited bandwidths and low design flexibility since the overlap between the optical field and the sensing species is nearly complete ${ }^{3}$.

In addition to the fraction of optical power available for sensing, the gas filling time is also an important parameter to consider when designing a fibre-based gas sensor since the microscopic size of the holes may lead by capillarity to an endless gas progression along the fibre. Previous papers monitored the gas filling process in PCFs using acetylene absorption lines and studied its dependence on fibre length ${ }^{3,4}$. Accurate expressions have been provided to predict the temporal response of a gas sensor ${ }^{5}$, but proved to be quite inconvenient due to the non-analytical nature of the solutions. This work aims at providing simple equations that can be applied to any type of fibre, fibre geometry or length. Our model for the filling time is based on the theory of gas diffusion where approximations have been made to mitigate the complexity of the equations. The validity of these approximations is verified by analyzing the gas filling dynamics in fibre samples of various geometries. Using this gas filling model, the filling time can be predicted for any given fibre geometry and length.

\section{GAS FILLING DYNAMICS}

This section presents the equations governing the diffusion dynamics of gas molecules inside capillaries. The increase of the pressure as a function of time during the gas filling process is ${ }^{6}$ :

$$
\frac{P(t)}{P_{0}}=1-\frac{8}{\pi^{2}} \sum_{j=1,3,5}^{\infty} \frac{1}{j^{2}} \exp \left\lfloor-\left(\frac{j \pi}{\xi L}\right)^{2} D t\right\rfloor
$$

*isabelle.dicaire@epfl.ch

Fourth European Workshop on Optical Fibre Sensors, edited by José Luís Santos, Brian Culshaw,

José Miguel López-Higuera, William N. MacPherson, Proc. of SPIE Vol. 7653, 76530L

(c) 2010 SPIE · CCC code: $0277-786 X / 10 / \$ 18 \cdot$ doi: $10.1117 / 12.866465$

Proc. of SPIE Vol. 7653 76530L-1 
where $L$ is the fibre length and $\xi$ is a geometrical factor equal to two for gas diffusing into a capillary from one end and equal to unity for gas diffusing from both ends. Theoretically Eq. 1 is valid only if the diffusion coefficient $D$ is constant during the gas filling process, which is not the case for pure gas diffusing in vacuumed microstructured fibres since it is pressure-dependent. As one would have to use numerical solutions ${ }^{5}$ to accurately describe the filling process, we will approximate the diffusion coefficient as being constant throughout the filling process and the parameter $D$ in Eq. 1 will represent the average filling speed for a given average pressure $\bar{P}$. It can be calculated using semi-empirical relations ${ }^{7}$ :

$$
D=\frac{a^{2} \bar{P}}{8 \eta}+Z \times \frac{2}{3} a \bar{v}, \quad \bar{v}=\sqrt{\frac{8 k_{B} T}{\pi m}},
$$

where $a$ is the capillary radius, $\eta$ is the viscosity of the gas, $\bar{v}$ is the mean molecular velocity, $k_{B}$ is the Boltzmann constant, $T$ is the temperature, $m$ is the molecular mass, and $Z$ is a scaling parameter which depends on the average pressure $\bar{P}$ via the mean free path $\lambda$ of the gas molecules:

$$
Z=\frac{1+2.507 a / \lambda}{1+3.095 a / \lambda}, \quad \lambda=\frac{k_{B} T}{\sqrt{2} \pi \bar{P} \delta^{2}},
$$

with $\delta$ being the diameter of the gas molecule. The filling time is then here defined as the time required for the pressure in the fibre to reach $85 \%$ of an asymptotic pressure $P_{0}$ (which can be reached only after an infinite time):

$$
P=85 \% P_{0} \text {. }
$$

With the help of Eq. 1, our analytical model for the filling time $t_{\text {fil }}$ of gaseous species inside microstructured fibres gives:

$$
t_{\text {ful }}=\frac{(\xi L)^{2}}{\pi^{2} D} \ln \left\lfloor\frac{8}{\pi^{2}} \frac{P_{0}}{P_{0}-P}\right\rfloor
$$

and can be applied to any fibre geometry or length. From an experimental point of view, the average pressure inside a fibre during gas filling can be retrieved by monitoring the decreasing intensity $I(t, v)$ of an absorption line as a function of time:

$$
\frac{P(t)}{P_{0}}=\frac{\int I(t, v) / I_{0}(v) d v}{\int I_{f l l}\left(t_{f l l}, v\right) / I_{0}(v) d v} .
$$

This is done in the following experimental sections, where experimental filling times for various fibre samples will be obtained and compared with the predicted ones.

\section{EXPERIMENTS \& RESULTS}

The various fibre samples were first spliced at one end to standard SMF-28 fibres using an FSM-40S Fujikura arc fusion splicer. A dispersion-compensating fibre (DCF) with a $\sim 4 \mu \mathrm{m}$ core diameter was inserted between the SMF-28 and the PCFs to better adapt the mode field diameters. The typical splice loss between the SMFs and DCFs is 1dB compared to $2-3 \mathrm{~dB}$ for splices between DCFs and PCFs. These concatenated fibres were then placed inside a sealed gas chamber and vacuum was maintained for 4-8 days with the help of a turbo vacuum pump to let air fully evacuate the cladding holes. Acetylene gas was then introduced inside the chamber and the filling process was monitored using the R7, P15, and P14 absorption lines by modulating the injection current of a distributed-feedback (DFB) laser diode so as to sweep the laser line across the absorption line. The wavelength information was retrieved with a Burleigh WA-1000 wavemeter. The filling characteristics of the three fibre samples can be found in table 1 for a filling pressure of $77 \mathrm{mbar}$.

Table 1. Results of the gas filling experiments.

\begin{tabular}{|cc|c|c|c|c|c|}
\hline Fibre & & Length & Hole diameter & Experimental filling time & Theoretical filling time & Accuracy \\
\hline \hline Solid-core F. & $9.2 \mathrm{~m}$ & $5.4 \mu \mathrm{m}$ & $20 \mathrm{~h} 06 \mathrm{~min}$ & $17 \mathrm{~h} 30 \mathrm{~min}$ & $87 \%$ \\
\hline Central-hole F. & $5.4 \mathrm{~m}$ & $3.4 \mu \mathrm{m}$ & $9 \mathrm{~h} 06 \mathrm{~min}$ & $10 \mathrm{~h} 06 \mathrm{~min}$ & $90 \%$ \\
\hline Suspended-core F. & $2.7 \mathrm{~m}$ & $11.8 \mu \mathrm{m}$ & $33 \mathrm{~min}$ & $35 \mathrm{~min}$ & $94 \%$ \\
\hline
\end{tabular}


As seen in Table 1, the temporal response of a fibre gas sensor can be predicted with at least $85 \%$ accuracy using our analytical model by calculating the corresponding diffusion coefficients. Moreover, Figure 2 illustrates the good agreement between the calculated diffusion coefficients $D$ and the data points for gas diffusion in suspended-core fibres. One should note that the uncertainty in the measured values is due to normalization difficulties related to the oscillating background pattern often seen in PCF spectra combined with the difficulties in evaluating the asymptotic pressure $P_{0}$.

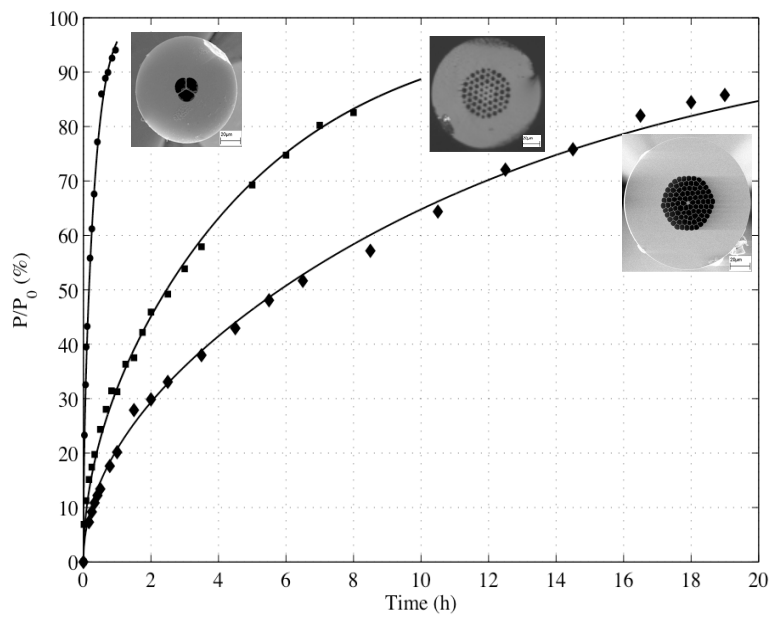

Figure 1. Measured average pressure as a function of time in a solid-core PCF (diamonds), a central-hole solid-core PCF (squares), and a suspended-core PCF (circles). The black curves represent nonlinear fittings of Eq. 1 from which experimental diffusion coefficients could be obtained.

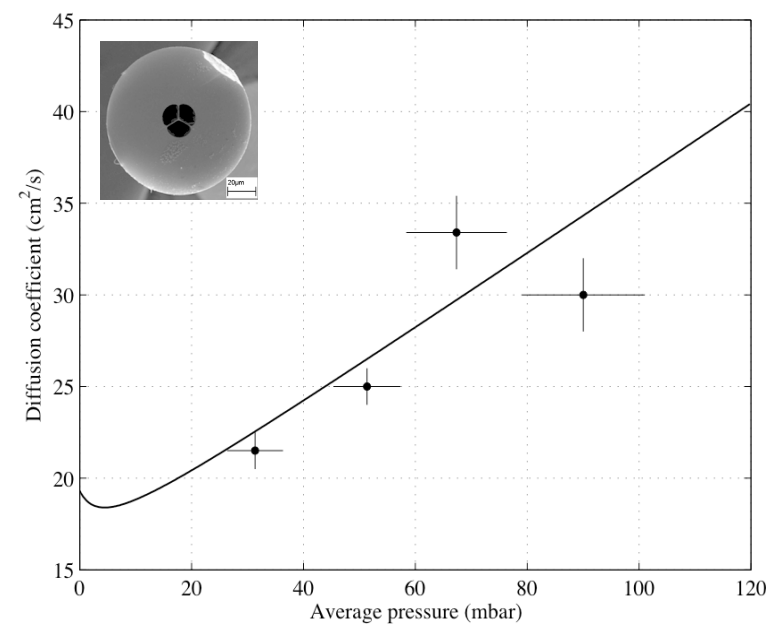

Figure 2. Calculated diffusion coefficient for acetylene gas diffusing in suspended-core fibres $(11.8 \mu \mathrm{m}$ hole diameter). The average pressures are taken as $2 / 3$ of the filling pressures with $10 \%$ uncertainty. The data points represent experimental diffusion coefficients obtained using Eq. 1.

\section{IDEAL GAS FILLING CONDITIONS}

Apart from being used as chemical sensors to detect hazardous gases, microstructured fibres can be filled with lowpressure gases to make all-fibre gas cells. As the linewidth of absorption lines depends on gas pressure, the fibres usually contain pure gas at pressures less than 200 mbar. However, when gas chambers or similar devices are used for fibre filling, the gas pressure inside the chamber will increase with time due to three main factors: virtual or real leaks, outgassing of molecules at low pressure or backstreaming from the vacuum pump ${ }^{8}$. As a result, the gas leakage rate together with long filling times will introduce impurities into the gas cell. Figure 3 presents the filling time in microstructured fibres for any combination of hole diameter and fibre length.

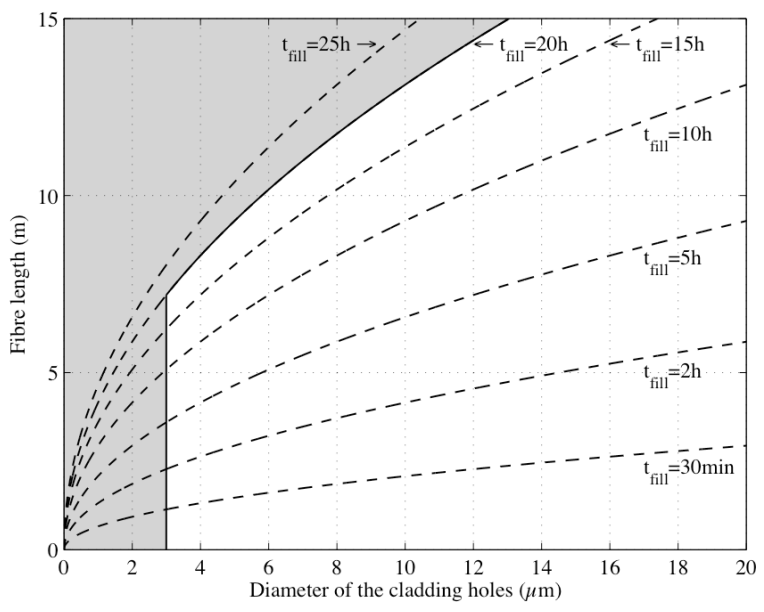

Figure 3. Contours of constant filling time are plotted in the $L-2 a$ plane according to Eq. $2 \& 5\left(\bar{P}=85 \% P_{0}\right)$. 
Limiting the filling time according to a few selected criteria then secures the efficiency of the gas cell. As the addition of impurities in the gas cell increases the Lorentzian absorption linewidth $\Delta v_{\mathrm{L}}$ of the Voigt profile according to the empirical relation:

$$
\Delta v_{v}=0.5346 \Delta v_{L}+\sqrt{0.2169 \Delta v_{L}^{2}+\Delta v_{G}{ }^{2}},
$$

where $\Delta v_{\mathrm{V}}$ and $\Delta v_{\mathrm{G}}$ are the Voigt and Gaussian linewidths respectively, allowing a maximum $5 \%$ linewidth broadening due to impurities will yield a maximum impurity percentage of $\sim 10 \%$ for contamination by air ${ }^{9}$. According to a measured gas leakage rate of $1 \mathrm{mbar} / \mathrm{h}$, this corresponds to a maximum allowed filling time of 10 hours for a $100 \mathrm{mbar}$ filling pressure. In addition, by considering minimum fibre lengths of $5 \mathrm{~m}$ to ensure a minimal absorption efficiency according to the Beer-Lambert absorption law, the fibre holes should have a minimum diameter of $3 \mu \mathrm{m}$. These restrictions for the fibre characteristics are illustrated as shaded regions in Fig. 3. This is another indication that the gas flow dynamics is a crucial matter when designing microstructured fibre for gas detection and all-fibre gas cell applications.

\section{CONCLUSION}

Helpful expressions predicting gas-filling times in photonic crystal fibres have been presented in this paper. Based on the theory of gas diffusion, our model can be readily applied to any fibre geometry or length as confirmed by the experimental results. By studying the gas filling process for various pressures inside a given fibre sample, we could experimentally confirm the pressure dependence of the diffusion coefficient and therefore the relation between filling pressure and filling time. Conversely, by monitoring the gas filling process in fibres, parameters such as the diameter of the cladding hole and the gas viscosity can be estimated. This study of the gas flow dynamics can be used as a metrological tool to characterize photonic crystal fibres and the gas species.

\section{ACKNOWLEDGMENTS}

The authors would like to thank University Marie-Curie Skłodowskiej (UMCS) in Lublin, Poland, for providing the suspended-core fibres in the framework of COST 299 Action "FIDES". They would like also to thank the Nanyang Technological University (NTU), Singapore and the Yonsei University in Seoul, South Korea, for providing the microstructured fibres. This work is financially supported by the European Space Agency (ESA) through Grant No. 20200/06/NL/PA and by the Swiss National Office for Education and Research through Project COST C06.0015.

\section{REFERENCES}

[1] Fini, J. M., "Microstructure fibres for optical sensing in gases and liquids," Measurement Science and Technology, 15(6), 1120-1128 (2004).

[2] Euser, T. G., Chen, J. S. Y., Scharrer, M., Russell, P. S. J., Farrer, N. J., and Sadler, P. J., "Quantitative broadband chemical sensing in air-suspended solid-core fibers," Journal of Applied Physics, 103(10), 103108-7 (2008).

[3] Ritari, T., Tuominen, J., Ludvigsen, H., Petersen, J., S- rensen, T., Hansen, T., and Simonsen, H., "Gas sensing using air-guiding photonic bandgap fibers," Opt. Express, 12(17), 4080-4087 (2004).

[4] Gayraud, N., Kornaszewski, u. W., Stone, J. M., Knight, J. C., Reid, D. T., Hand, D. P., and MacPherson, W. N., "Mid-infrared gas sensing using a photonic bandgap fiber," Appl. Opt., 47(9), 1269-1277 (2008).

[5] Henningsen, J., and Hald, J., "Dynamics of gas flow in hollow core photonic bandgap fibers," Appl. Opt., 47(15), 2790-2797 (2008).

[6] Jost, W., [Diffusion in solids, liquids, gases] Academic Press, New York (1970).

[7] Dushman, S., and Lafferty, J. M., [Scientific Foundations of Vacuum Techniques] John Wiley \& Sons, New York (1962).

[8] O'Hanlon, J. F., [A user's guide to vacuum technology] Wiley, New York, 536 p. (2003).

[9] Bond, K. S., Collett, N. D., Fuller, E. P., Hardwick, J. L., Hinds, E. E., Keiber, T. W., Kelly-Morgan, I. S. G., Matthys, C. M., Pilkenton, M. J., Sinclair, K. W., and Taylor, A. A., "Temperature dependence of pressure broadening and shifts of acetylene at 1550nm by He, Ne, and Ar," Applied Physics B: Lasers and Optics, 90(2), 255-262 (2008). 